\title{
Whole exome sequencing reveals a combination of rare high and low penetrance variants that correlates with familial breast cancer relative risk
}

Mariem Ben rekaya ( $\nabla$ rekayamariem@gmail.com )

Université de Tunis El Manar Faculté de Médecine de Tunis, Theranostic Biomarkers in cancers, UR17ES15 https://orcid.org/0000-0001-7176-5201

Yosr Hamdi

Laboratory of Biomedical Genomics and Oncogenetics (LR16IPT05), Institut Pasteur de Tunis, University of Tunis El Manar, El Manar I, 2092 Tunis, Tunisia ;

Laboratory of Human and Experimental Pathology, Institut Pasteur de Tunis, Tunis, Tunisia

Houda El Benna

Laboratory of Biomedical Genomics and Oncogenetics (LR16IPT05), Institut Pasteur de Tunis, University of Tunis El Manar, El Manar I, 2092 Tunis, Tunisia ;

Department of Medical Oncology, Abderrahmane Mami Hospital, 2080 Ariana, Tunisia

\section{Nessrine Mejri}

Laboratory of Biomedical Genomics and Oncogenetics (LR16IPT05), Institut Pasteur de Tunis, University of Tunis El Manar, El Manar I, 2092 Tunis, Tunisia ; Department of Medical Oncology, Abderrahmane Mami Hospital, 2080 Ariana, Tunisia

Olfa Jaidane

Surgical oncology department, Institute Salah Azaiez of Oncology, Boulevard 9 avril 1938 Beb Saadoun, 1006 Tunis, Tunisia

Jihene Ayari

Medical Oncology Service, Military Hospital of instruction of Tunis, Mont Fleury-1008 Tunis, Tunisia

\section{Sonia Ben Nasr}

Laboratory of Biomedical Genomics and Oncogenetics (LR16IPT05), Institut Pasteur de Tunis, University of Tunis El Manar, El Manar I, 2092 Tunis, Tunisia ; Medical Oncology Service, Military Hospital of instruction of Tunis, Mont Fleury-1008 Tunis, Tunisia

Hamza Dallali

Laboratory of Biomedical Genomics and Oncogenetics (LR16IPT05), Institut Pasteur de Tunis, University of Tunis El Manar, El Manar I, 2092 Tunis, Tunisia

Olfa Messaoud

Laboratory of Biomedical Genomics and Oncogenetics (LR16IPT05), Institut Pasteur de Tunis, University of Tunis El Manar, El Manar I, 2092 Tunis, Tunisia

Rym Meddeb

5Department of Hereditary and Congenital Disorders, Charles Nicolle Hospital, Tunis, Tunisia

Najah Mighri

Laboratory of Biomedical Genomics and Oncogenetics (LR16IPT05), Institut Pasteur de Tunis, University of Tunis El Manar, El Manar I, 2092 Tunis, Tunisia

Maroua Boujemaa

Laboratory of Biomedical Genomics and Oncogenetics (LR16IPT05), Institut Pasteur de Tunis, University of Tunis El Manar, El Manar I, 2092 Tunis, Tunisia

\section{Mohamed Samir Boubaker}

Laboratory of Biomedical Genomics and Oncogenetics (LR16IPT05), Institut Pasteur de Tunis, University of Tunis El Manar, El Manar I, 2092 Tunis, Tunisia ;

Laboratory of Human and Experimental Pathology, Institut Pasteur de Tunis, Tunis, Tunisia

\section{Abderazek Haddaoui}

Medical Oncology Service, Military Hospital of instruction of Tunis, Mont Fleury-1008 Tunis, Tunisia

\section{Ridha Mrad}

Department of Hereditary and Congenital Disorders, Charles Nicolle Hospital, Tunis, Tunisia

\section{Hamouda Boussen}

Department of Medical Oncology, Abderrahmane Mami Hospital, 2080 Ariana, Tunisia

Sonia Abdelhak

Laboratory of Biomedical Genomics and Oncogenetics (LR16IPT05), Institut Pasteur de Tunis, University of Tunis El Manar, El Manar I, 2092 Tunis, Tunisia Soumaya Labidi

Laboratory of Biomedical Genomics and Oncogenetics (LR16IPT05), Institut Pasteur de Tunis, University of Tunis El Manar, El Manar I, 2092 Tunis, Tunisia ; Department of Medical Oncology, Abderrahmane Mami Hospital, 2080 Ariana, Tunisia

\section{Research}

Keywords: OGG1, BRCA2, polygenic inheritance, rare variants, BER

Posted Date: July 17 th, 2020

DOI: https://doi.org/10.21203/rs.3.rs-22989/v2

License: (c) (i) This work is licensed under a Creative Commons Attribution 4.0 International License. Read Full License 


\section{Abstract}

Background: Genetic risk factors of breast cancer are very heterogeneous and complex. They vary according to the familial relative risk, the age of cancer diagnosis of the index case and the age of the affected relatives.

Objectives: We aimed to investigate and identify simultaneously all rare pathogenic and common variants in unrelated BC cases with different relative risk ratios for breast cancer and evaluate the contribution of these variants in genetic susceptibility to breast cancer.

Patients and Methods: All frequent mutations in BRCA genes previously identified in Tunisia have been excluded by Sanger sequencing in 42 women affected with high family risk having at least 3 cancer affected related individuals. Two unrelated cases having two different family histories (in terms of different numbers of affected first-degree relatives and young age onset) have been selected for whole exome sequencing. The first family is composed of three sisters F1.1, F1.2 and F1.3 affected at 46, 50, and 32 years old, respectively. The second has only two breast cancer cases, F2.2 and F2.4, affected at late age 61 and 70 years old, respectively, in addition to other 5 members affected by different kinds of cancer. Selected high risk variants were confirmed and segregation analysis was performed using Sanger sequencing.

Results and discussion: For F1.1 case, we identified a pathogenic frame-shift loss of function variant in BRCA2 p.Val1283Lysfs. For F2.2 we identified a pathogenic rare variant in OGG1, p.Arg46GIn that co-segregates with a rare non sense variant in BRCA2 p.K3326X, only in the breast cancer affected cases. Moreover, F2.2 patient has 9 other common low penetrant variants in different loci known to represent independently minor, but cumulatively significant, increased risk for breast cancer.

Conclusion: Family history and the young age at onset for patient F1.1 correlate with the presence of a rare high penetrant variant (p.Val1283Lysfs) in BRCA2 gene. However, the late age at onset and the less severe phenotype for patient F2.2 are probably the consequence of the presence of a pathogenic variant p.Arg46GIn in OGG1 gene that co-segregate with a low penetrant variant Lys3326X in BRCA2 only in breast cancer cases.

\section{Background}

Cancer is one of the leading causes of death world-wide. There were 6.7 million new cancer cases and 3.5 million deaths among females worldwide in 2012. Among them, $56 \%$ of cases and $64 \%$ of deaths were in under-developed countries. These numbers are expected to increase to 9.9 million cases and 5.5 million deaths among females annually by 2030 as a result of the lifestyle changes and aging of the populations ${ }^{1}$.

Breast cancer (BC) is the most frequently diagnosed cancer worldwide especially in economically developed countries. In Tunisia, BC is a major public health problem with at least 2300 new cases per year. Many studies suggest that it is more aggressive than in Western countries, with notably large proportions of young patients ${ }^{2}$

Both non-genetic and genetic factors are involved in the etiology of breast cancer. About $15 \%$ of cases exhibit a family history of the disease which represents the strongest risk factor for mutation carriers. Its risk varies according to the mutation location and the genes involved. Therefore, genetic counseling should incorporate both family history profiles and mutation location ${ }^{3}$. A measure of this familial clustering is the familial relative risk (FRR), defined as the ratio of the risk of disease for a relative of an affected individual to that for the general population. Current International guidelines for BRCA testing use (i) breast or ovarian family, (history, (ii) young age at diagnosis $\leq 36$ years and (iii) triple-negative breast cancer as the most risk factors. However, the commonly used guidelines for testing were insufficient to detect all mutation carriers in the $\mathrm{BC}$ cohorts ${ }^{4}$. Indeed, a higher rate of both $B R C A 1$ and $B R C A 2$ mutations has been observed in affected patients from North Africa without family history $(8.0 \%$ in North Africa versus $1.1 \%$ in France for $B R C A 1$ mutations, $\mathrm{P}=0.02 ; 7.2 \%$ in North Africa vs. $1.1 \%$ in France for BRCA2 mutations:, $\mathrm{P}<0.05)^{5}$.

The genetic variants associated with breast cancer risk can be classified as high-penetrant mutations that are rare in the population but associated with a very high risk (relative risk of carriers versus non carriers of 5 to $>20$ ); moderate penetrant variants associated with a moderate risk ( $5>$ relative risk $>1.5$ ) ; and lowpenetrant polymorphisms which are common and associated with a small risk (relative risk <1.5) 6 .

At the age of 80 , cumulative cancer risk for $B R C A 1$ and $B R C A 2$ mutation carriers ranges from $69 \%$ to $72 \%$ for breast cancer development, and from $17 \%$ to $44 \%$ for ovarian cancer ${ }^{3}$. This variability is explained by other genetic modifiers and/or environmental factors ${ }^{7}$. Studies of the genetic variants influencing the risk of breast cancer in BRCA1/2 mutation carriers have been conducted first by the Consortium of Investigators of Modifiers of $B R C A 1$ and $B R C A 2$ (CIMBA) and then by the Collaborative Oncological Gene-environment Study (COGS). More than one hundred SNPs have been so far identified that are associated with the risk of developing breast or ovarian cancer for $B R C A 1$ or $B R C A 2$ carriers ${ }^{8-13}$. More recent studies have shown that common variants in genes involved in DNA repair pathways especially Base Excision Repair (BER) have a synergistic functional effect increasing cancer risk susceptibility in $B R C A 2$ mutation carriers ${ }^{14,15}$. Among 144 SNPs analyzed in a two stage study involving 23,463 carriers from the CIMBA consortium, eleven SNPs showed evidence of association with breast and/or ovarian cancer at $p<0.05$ in the combined analysis. Four of the five genes for which strong evidence of association was observed were DNA glycosylases especially, OGG1, TDG and NEIL2. Most of these SNPs are common and non-coding, present in regulatory regions. Unfortunately, we have no idea on the role of rare coding modifiers variants located in these genes.

In another side, a large proportion of all breast cancers arises in a genetically susceptible minority of women that are not carriers of $B R C A 1$ or $B R C A 2$. Susceptibility to breast cancer is likely to be the result of at risk alleles in many different genes. Previous studies suggested that disease susceptibility in non carriers of $B R C A 1 / 2$ mutations is explainable, in a polygenic model, by large numbers of susceptibility polymorphisms that are multiplicatively acting on risk ${ }^{16-18}$. It is estimated that $28 \%$ of familial breast cancer risk is explained by common breast cancer susceptibility loci. In some cases, SNP associations may be 
specific to some ethnicity or estrogen receptor ${ }^{13}$. The most recent and largest breast cancer Genome Wide Association study (GWAS) using the Illumina OncoArray BeadChip has identified a total of 172 risk-associated SNPs that account for an estimated $\sim 18 \%$ of familial relative risk ${ }^{19-21}$.

As focusing on common non coding variants, GWAS studies have limited the capacity to identify interactions between $B R C A 1$ and $B R C A 2$ mutations and rare coding modifiers variants ${ }^{22}$. A portion of missing heritability in familial breast cancer is likely represented by rare functional coding variants in genes not currently present on available panels ${ }^{23}$. These rare coding variants could be identified using Whole Exome Sequencing (WES) or Multi Genes High Throughput Sequencing.

Recently, WES technology has been demonstrated to be efficient in the discovery of novel breast cancer predisposition genes such as those that encode proteins involved in the DNA damage response or DNA repair ${ }^{24}$ and has helped to determine the frequency of causal germline mutations ${ }^{23,25}$ and to identify novel possible genetic modifiers of risk for early-onset breast cancer predisposition in carriers of high-risk mutations ${ }^{26}$.

In the present study, we used WES to explore potential rare pathogenic variants for two cases having different relative risk ratios for breast cancer and evaluate the contribution of these variants in the genetic susceptibility to the disease.

\section{Patients And Methods}

\section{- Clinical manifestations and family history}

This study was conducted according to the principles of the declaration of Helsinki and has obtained the ethics approval from the institutional review board of Pasteur Institute of Tunisia Registration number 2017/16/E/hospital a-m/V1. Forty two breast cancer unrelated affected patients were recruited from the Oncology Department of Abderrahman Mami Hospital of Ariana or from the surgical oncology department of Salah Azaiez Institute, or the Oncology department of Military hospital of Tunis.

Clinical and epidemiological characteristics of the available Breast cancer affected members are summarized in Table 1. The first patient, F1.1, diagnosed at the age of 46 years, has two affected sisters (F1.1 and F1.3 ) diagnosed respectively at the age of 50 and 32 years. The second patient, F2.2, diagnosed at 61 years, has a sister, F2.4, affected by breast cancer at 71 years, another sister, F2.1, affected by colorectal cancer and diagnosed at 66 years, a third sister affected by cervical cancer, one brother ,F2.5, affected by testicular cancer diagnosed at 60 years and the father who died of Larynx cancer at age of 49 in addition to one related individual who died of colorectal cancer.

\section{- Exome sequencing}

Whole-exome sequencing was performed for patients F1.1 and F2.2. Exome was captured from genomic DNA using Agilent SureSelect Protocol Version 1.2 (Agilent Technologies; Santa Clara, CA, USA) and then sequenced on an Illumina HiSeq 2000 sequencer. We used BWA to align sequence reads to the hi19 reference genome and GATK to call SNVs and indels. Control quality showed that $88 \%$ of targeted bases were covered at $>20 \mathrm{X}$.

\section{- Exome sequencing data analysis}

The results were analyzed using the VarAft software version 1.6, (http://varaft.eu/index.php). For exome analysis, dominant models of inheritance have been selected. Given the number of variants identified in WES, and in order to prioritize them, variants were filtered according to several stringent criteria. Indeed, we kept only rare functional variants (missense, nonsense, splice site variants, and indels) that were heterozygous in the index cases and we discarded variants with a Minor Allele Frequency (MAF) $\geq 1 \%$ according to 4 databases ( 1000 Genomes Project, Exome Variant Server (EVS), Exome Aggregation Consortium (ExAC) and a local database encompassing 48 exomes of Tunisian individuals with no personal nor familial breast cancer history). We also excluded variants with low sequencing quality. A number of online tools were used to predict the functional impact and pathogenicity of the missense variants such as Mutation Taster, PolyPhen, SIFT. Of the variants that met these criteria, we selected all rare coding variants (frequency $<0.01$ ) described at least once as pathogenic in ClinVar and located in a gene that matched with breast cancer disorders according to the VarElect prioritization tool (http://varelect.genecards.org) ${ }^{27}$. We also extracted common at risk variants previously reported to contribute in increasing breast cancer risk.

\section{- Variant validations and co-segregation}

Exons with a bad coverage (i.e. Exon 5 in BRCA2 and Exon 13 in BRCA1) as well as exons 11 and 27 in BRCA2 and exon1 in OGG1 gene, which contain potential at risk variants, were amplified and sequenced by Sanger sequencing using the primers listed in (Supplementary Table 1) in order to confirm the variations and to analyze co-segregation for available related affected and healthy individuals.

\section{Results}

\section{Exome Analysis and interpretation strategies}

\subsection{Screening for variants in BRCA genes}

All heterozygous and homozygous variants in BRCA1 and BRCA2 genes, that have been identified in the two cases, are listed in Table 3 . The structure of the genetic profile between the two patients showed significant difference in terms of frequency and function (Fig1).

The patient F1.1 has 6 variants in BRCA1 and 6 variants in BRCA2. Among them, two interesting variants: the frame-shift deletion c.3847_3848delGT (p.Val1283Lysfs) (BIC: 4075delGT), classified as rare pathogenic variant rs80359405 and the frequent variant rs799905 predicted putative functional 
according to regulomdb software (Score=2b).

However for the patient F2.2, she has 16 variants in BRCA1 and 11 variants in BRCA2. None of them, is known, as a pathogenic variant. However, in silico analysis showed the presence of 4 frequent regulatory functional variants in BRCA1 gene (rs16940, rs3092994, rs1060915, rs3765640) that are responsible for cis regulation expression according to Encode data and RegulomeDB software (Score=1F). In addition, she has the rare non sense coding variant rs11571833, c.9976A>T; p.Lys3326Ter, that results in a 92 amino acid truncation of BRCA2 protein. This rare variant and other 3 frequent non synonymous variants (rs16942, rs1799966, rs144848) are classified as low penetrant breast cancer variants and could together generate a polygenic risk score (PRS) ${ }^{28}$ (Supplementary Table 2).

\subsection{Screening for rare and pathogen variants}

For the patient F1.1, we found one rare variant classified as pathogenic in ClinVar rs746229647/ rs80359405 in BRCA2. This gene has the highest Varelect Score 920,64 matching with the breast cancer disease. In addition, she has four rare missense variants at heterozygous state (rs1801155 APC c.3920T>A; p.I1307K; rs7418956 SPTA1:c.2373C>A, p.D791E; rs41298442 GCH1 c.671A>G p.K224R; rs184394424 FREM1 c.1493G>A, p.R498Q) classified as conflicting interpretations of pathogenicity and located in different genes having variable Varelect Score ranging from 670,03 to 14,31 (Table 2).

The variant, c.3846_3847del in BRCA2, was previously described as deleterious and associated with the hereditary cancer-predisposing syndrome. It is a frame-shift deletion c.3847_3848delGT (p.Val1283Lysfs) (BIC: 4075delGT), described to be causing breast cancer in males among Finns and associated with the young form of prostate cancer and colorectal cancer ${ }^{29}$. This mutation, classified as a breast cancer high penetrant variant, is found in $5 \%$ of $B R C A 2$ positive in Danish families ${ }^{30}$ and is one of the four founder mutations in BRCA2 gene in Norway ${ }^{31}$ but it has never been described among African populations.

The APC gene has the highest Varelect score $(670,03)$. The APC variant, c.3920T>A, is predicted as damaging by two in silico prediction softwares. It is described as a functional variant converting the DNA sequence to a homo-polymer region (A8) that is genetically unstable and prone to somatic mutation ${ }^{32}$. Previous studies have shown that it could predict the prevalence of breast, lung, urologic, pancreatic, and skin cancers ${ }^{33,34}$ and it has been associated with an increased risk of colorectal cancer among Ashkenazi Jewish, Croatian, and Egyptian patients ${ }^{35-37}$.

For the variant in the SPTA1 gene (Score=33,86), it is associated with the elliptocytosis disease which is a heterogeneous red blood cell (RBC) membrane disorder. The gene encodes for an actin crosslinking and molecular scaffold protein. According to the My Genome Cancer database, somatic missense mutations in this gene are observed in cancers such as esophageal, genital tract, and endometrial. This mutation has been previously found in a Tunisian family and recently in two other asymptomatic patients but having ektacytometry profile consistent with mild hereditary elliptocytosis; this phenotype called "Jendouba spectrine phenotype" 38,39 .

For the two variants, GCH1 c.671A>G:p.K224R and FREM1 c.1493G>A; p.R498Q, none relevant data have been described in relation with cancer. The first gene is associated with the autosomal recessive Dystonia dopa responsive with or without hyperphenylalaninemia disease and is associated with an increased risk for Parkinson's disease ${ }^{40}$. The second gene is associated with the autosomal dominant Trigonocephaly_2 phenotype with nonsyndromic metopic craniosynostosis.

For patient F2.2, we have identified two rare pathogenic variants in OGG1 and GCGR genes, rs104893751 c.137G>A, p.R46Q and rs1801483 c.118G>A, p.G40S respectively and one variant in FTCD gene (rs35208133/rs398124234 c.990dupG, p.P331fs) classified as conflicting interpretation of pathogenicity.

The OGG1 gene has the highest Vareclect score matching with breast cancer disorders $(236,97)$. The variant R46Q has been previously described as a risk allele for the Human clear_cell_carcinoma_of_kidney that impairs the enzymatic activity of the OGG1 DNA glycosylase ${ }^{41}$ and recently observed in an affected member by a familial form of small intestinal neuroendocrine tumors (SI-NETs) and also in a putative clinically healthy carrier member 42 .

The second variant c.118G>A; p.G40S is present in the GCGR gene that encodes for the Glucagon Receptor. It has been associated with Type 2 diabetes in various white European populations ${ }^{43}$ and with hypertension in both European whites and Australians ${ }^{44,45}$. Interestingly, a recent study has provided experimental evidence that hyperglucagonemia in type 2 diabetes promotes colon cancer progression via GCGR-mediated regulation of AMPK and MAPK pathways ${ }^{46}$. It is known that women with diabetes mellitus are at higher risk of breast cancer-specific and all-cause mortality after initial breast cancer diagnosis ${ }^{47}$. So we hypothesize that this variant could have increased the risk of breast cancer in our case.

For the third variant, c.990dupG: p.P331fs on FTCD gene. Mutations in FTCD represent the molecular basis for the mild phenotype of the Glutamate form iminotransferase deficiency, an autosomal recessive disorder and the second most common inborn error of folate metabolism. There is a conflicting epidemiological evidence on the role of folate in breast cancer risk. A recent metanalysis review has shown that breast cancer does not appear to be associated with folate intake, and this did not vary by menopausal status or hormonal receptor status. In addition, folate blood levels also do not appear to be associated with breast cancer risk ${ }^{48}$

\subsection{Screening for Common at risk Variants}

Investigation of common at risk variants could contribute to estimate and refine each individual risk and help to identify the highest risk patient. For this, among a published list of 182 risk associated SNPs that have displayed genome-wide significant associations with breast cancer ${ }^{13,21}$, we have extracted those present in each of our patients. 
For the patient F1.1, we found only one SNP rs11374964, however for the patient F2.2, we found 6 SNPs (rs2992756, rs4971059, rs4245739, rs6964587, rs11374964, rs2236007) (Supplementary Table 3). These six SNPs added to the four variants that are present in BRCA genes could contribute together to increase the individual risk for developing breast cancer.

\section{Sanger confirmation and validation}

The BRCA2 c.3847_3848delGT frame-shift mutation was confirmed by Sanger sequencing and co-segregation analysis was performed in the two other affected sisters (F1.2 and F1.3). However, It was absent in 40 available affected with high family risk women having at least 3 affected related individuals.

We also confirmed that the second variant in BRCA2 gene, c.9976A>T; p.Lys3326Ter, was present in the index case and also in her BC affected sister (F2.4) but absent in the healthy sister (F2.3) and brother (F2.6) and also in the affected colorectal cancer sister (F2.1) and the affected testicular cancer brother (F2.5). Thus, confirming that this variant segregate only with the breast cancer phenotype.

For the OGG1 variant, it was confirmed by Sanger sequencing and co-segregation analysis was performed. It was found at a heterozygous state in the two BC affected patients (F2-2 and F2-4) and in one healthy sister F2-3 and absent in the 3 remaining family members. F2-3 has 68 years old, she reached menopause at the age of 48 years old. Cosegregation of the two variants in BRCA2 and OGG1 was observed only in the two BC affected cases, suggesting an additive risk.

\section{Discussion}

The identification of mutations responsible for breast cancer through clinical genetic testing enables patients to benefit from early screening and prevention strategies, some of which provide generally survival benefit. Using next generation sequencing allowed the identification of all rare and common variants that could be linked to breast cancer predisposition.

According to the international guideline for BRCA testing, female members in F1 family should undertake BRCA test because of the positive family history and the young age at onset for the third sister F1.3 (31 years old) (Table 1). Results of WES showed a highly deleterious variant c.3847_3848delGT in BRCA2 gene. This mutation has been described as founder and frequent in the Danish population ${ }^{49}$. It is also present among Japanese patients and other Asian populations but it is rare elsewhere ${ }^{50}$. The index case has, in addition, a variant, lle1307Lys in APC gene, previously described as a risk factor for breast, lung, urologic, pancreatic, and skin cancers ${ }^{33,34}$ and has been associated with an increased risk of colorectal cancer among Ashkenazi Jewish, Croatian, and Egyptian patients ${ }^{35-37}$. This gene encodes a tumor suppressor protein that acts as an antagonist of the Wnt signaling pathway that regulates crucial aspects of cell fate determination, cell migration, cell polarity, neural patterning and organogenesis during embryonic development. This protein can modulate the BER pathway through an interaction with the DNA polymerase $\beta$ (Pol- $\beta$ ) and the flap endonuclease 1 (Fen- 1 ) and consequently might play an important role in carcinogenesis and chemotherapy by determining whether cells with DNA damage survive or undergo apoptosis ${ }^{51,52}$. In breast tumors, the transcriptional silencing of the $A P C$ gene by promoter hypermethylation has been detected in up to $70 \%$ of inflammatory human breast tumors ${ }^{53}$ and $7 \%$ of metaplastic breast carcinomas ${ }^{54}$. In addition, screening of $A P C$ and Fen 1 polymorphic and/or mutational variations could provide an important tool in the assessment of individual DNA repair capability and the risk for breast cancer development ${ }^{51}$. It is also suggested that APC selectively mediates response of chemotherapeutic agents in breast cancer. Since cisplatin and doxorubicin cause oxidative DNA damage, the chemotherapeutic resistance of the MMTVPyMT/ApcMin+ mammary tumors could also be due to increased BER because mutant APC have a decreased DNA damage activity which enhances cell survival ${ }^{55,56}$. Furthermore, we have identified 3 other conflicting pathogen variants in three different genes, SPTA1, GCH1, FREM1, with unknown role in the tumorigenesis process neither a relationship with the breast cancer disorders.

For the F2.2 patient and her affected sister, they have a late onset age ranging from 62 to 70 years with non aggressive tumor according to histopathology test. They responded well to treatment without signs of recidivism. We found a rare pathogen variant, R46Q OGG1, in the two affected sisters and also in the clinically healthy sister who is currently 73 years. This variant has been previously described as a risk allele for the Human clear_cell_carcinoma_of_kidney that impairs the enzymatic activity of the OGG1 DNA glycosylase ${ }^{41}$. R46Q OGG1 has been recently observed in a patient with a familial form of SI-NETs and also in a putative clinically healthy carrier member ${ }^{42}$.

In addition, significant associations between other OGG1 germline variants and breast cancer risk have been shown by meta-analysis and experimental data. For some missense variants in OGG1, the risk increases by 14 -fold $(\mathrm{p}<0.01)$ and reach 18 -fold $(\mathrm{p}<0.004)$ in breast cancer patients compared with controls 57 . Also, some common regulatory variants in OGG1 and other DNA glycosidase like NEIL2 are classified as potential cancer risk modifiers for BRCA1 and BRCA2 mutations carriers because they exert a synergetic effect with BRCA mutations on DNA damage and telomere shortening ${ }^{15,58}$.

For this patient, we have identified a rare non sense low penetrant variant in $B R C A 2$ gene, p.K3326X, combined with 4 common regulatory functional variants in BRCA1 gene (rs16940, rs3092994, rs1060915, rs3765640) predicted to have a functional role for cis-regulation expression and also 9 other common at risk SNPs : rs144848, rs1799966, rs1042522, , rs2992756, rs4971059, rs4245739, rs6964587, rs11374964, rs2236007 respectively in genes BRCA2, BRCA1, TP53, and loci 1p36.13, 1q22, 1q32.1, 7q21.2, 11q22.3, 14q13.3, reported to represent independently minor, but cumulatively significant, increased risk for breast cancer $^{13}, 19-21,28$

For the variant $\mathrm{K} 3326 \mathrm{X}$, it has been previously found in linkage disequilibrium with the variant rs $144848, B R C A 2 \mathrm{p} . \mathrm{N} 372 \mathrm{H}$, in $32 \mathrm{patients}$ sharing the same ancestor haplotype ${ }^{59}$. These two variants are present in our patient suggesting that they could be in linkage disequilibrium. A recent metanalysis study reveals that the rs $144848 \mathrm{H}$ allele could be a low-penetrant risk factor enhancing carcinogenesis in breast cancer ${ }^{60}$. The K3326X, which co-segregates in some cases with other deleterious BRCA2 mutations, is described as a low penetrant variant associated with a modestly high risk of breast cancer 59,61 . This 
variant confers susceptibility to multi-organ cancers: ovarian, breast, larynx and bladder (Supplementary Table 4) ${ }^{62-64}$. This is consistent with the family history of our patient that reveals that her father died of larynx cancer at 49 years old. Unfortunately, we could not verify if he was carrying this variation. Functional effects of the rs 11571833 have been reported in different studies, e.g. it is classified pathogenic class 5 according to the in vitro splicing assays ${ }^{65}$. Indeed, the $B R C A 2 \mathrm{COOH}$ terminus interacts with Rad51. Hence, homozygous germ-line deletion of BRCA2 exon 27 disrupts homologous recombinationmediated DNA repair and results in hypersensitivity to ionizing radiation and rapid senescence. ${ }^{66,67}$. A recent study has shown that the K3326X acts as a trans-eQTL involved in DNA repair pathway. In addition, it exhibits statistically significant association with expression of TRPC6 gene and $4 \mathrm{q} 21$ locus ${ }^{68}$. The $4 q 21$ has been recently identified as a novel breast cancer susceptibility locus associated with differential allelic expression ${ }^{69}$. This locus has been identified among the most frequent candidate loci with at high risk haplotype (haplotype frequency $>5 \%$ ), through a genome wide haplotype study in the general Tunisian population ${ }^{70}$. Little is known about the involvement of common variants and their association with breast cancer risk in Tunisia.

The "rare variant hypothesis" for susceptibility to common diseases postulates that a significant proportion of the inherited component might be due to the addition of the effects of a series of low frequency and independently acting variants from a variety of genes, each conferring a moderate but detectable increase in the relative risk ${ }^{71}$. Accumulation of rare genetic variants in DNA repair genes probably increases the deleterious effect of mutations in the high penetrant $B R C A$ genes by their ability to weaken response of the DNA repair system to oxidative damage. Actually, most of them are often referred as unclassified variants with uncertain clinical significance, thus creating a serious challenge to genetic testing. Familial segregation analysis, functional studies and in vitro assays could help to better assess analytical and clinical interpretation of these variants ${ }^{72,73}$.

Our results join those of previous studies and support the suggestion of screening variants in DNA repair genes for $B R C A 2$ carriers of low or high penetrant variations. This investigation allows the assessment of the individual DNA repair capacity and help to refine the breast cancer risk. At risk individuals who are carriers of functional variants in DNA repair genes might lower their breast cancer risk by reducing exposure to environmental carcinogens ${ }^{51}$.

Combination of rare pathogen variants in DNA repair genes with high or low penetrant variants in BRCA genes among Tunisian breast cancer cases might explain the relatively young onset age and the aggressive tumor types observed in some cases described in local epidemiological reports. Our previous studies have shown a relatively high rate of consanguinity 74,75 that increases the frequency of monogenic diseases such autosomal recessive DNA repair disorders predisposing to cancer and raise the prevalence of healthy carriers ${ }^{76,77}$; (Ben Rekaya unpublished data).

In summary, using WES and segregation analysis, we have identified a low penetrant variant, $\mathrm{K} 3326 \mathrm{X}$ in $B R C A 2$ gene, that co-segregates with a rare pathogenic variant, R46Q in OGG1 gene, only in breast cancer affected cases. The OGG1 variation is a candidate risk factor predisposing to the disease. In addition, other rare variants such as the variant in GCGR gene should be investigated in future studies to understand their potential role. It is also recommended that the ten risk common variants found in $B R C A$ genes and in others candidate loci be investigated through a large association study to better understand their (synergic) implication in the disease. For the F1 family, all affected members have benefited from a specific oncogenetic counseling. Further investigation should be considered to calculate its frequency in North African population.

\section{Conclusion}

WES has been successful in identifying rare coding variants involved in breast cancer etiology. There is a strong evidence that rare variants in DNA repair gene have an important role in breast cancer genetic background. These variants although individually rare, are collectively frequent, and even though their effect size are greater than those observed for common variants. Most large studies should be considered to confirm the role and the interaction between these rare variants in breast cancer genetic etiology.

\section{List Of Abbreviations}

Base Excision Repair BER

BC Breast cancer

Consortium of Investigators of Modifiers of BRCA1 and BRCA2

CIMBA

Collaborative Oncological Gene-environment Study COGS.

DNA polymerase $\beta \quad$ Pol- $\beta$

Exome Aggregation Consortium ExAC

Exome Variant Server EVS

Flap Endonuclease $1 \quad$ Fen-1

Familial Relative Risk $\quad$ FRR

Genome Wide Association study GWAS

Red Blood Cell $\quad$ RBC

Small Intestinal Neuroendocrine Tumors SI-NETs 


\section{Declarations}

Ethics approval and consent to participate: Written informed consents were obtained from all participants. Ethical approval according to the Declaration of Helsinki Principles was obtained from the biomedical ethics committee of Institut Pasteur de Tunis (2017/16/E/hospital a-m/V1).

Competing interests: The authors declare that they have no competing interests.

\section{Authors' contributions}

Mariem Ben Rekaya (MBR), Yosr Hamdi (YH), Houda El Benna (HEB), Nessrine Mejri (NM), Olfa Jaidane(OJ), Jihene Ayari(JA), Sonia Ben Nasr (SBN), Hamza Dallali(HD), Olfa Messaoud (OM), Rym Meddeb(RM), Najah Mighri (NMI), Maroua Boujemaa(MB), Abderazek Haddaoui(AH), Ridha Mrad(RMD), Hamouda Boussen(HB), Sonia Abdelhak(SA), Soumaya Labidi (SL).

Study conception and design: MBR, HB, SA and SL; Data acquisition: MBR, YH, HEB, SL, NM, OJ, JA, SBN, NMI, MB; Analysis and interpretation of molecular data: MBR, YH; Analysis and interpretation of the patient clinic-pathological data: OJ, HEB, SL, JA SBN, RM, and NM; Bioinformatic analysis: HD, MBR, YH. Contribution to the interpretation of the results : HB, SA, SL, NJ, and YH Technical experiment: MBR, OM, YH. Drafting of the full article: MBR; Editing and critical revision of the manuscript: SA, YH, SL, NM, OJ, JA, SBN, RM, RMD, AH, HB, and OM. Submission: MBR All authors read and approved the final manuscript.

\section{Availability of data and materials}

All data generated or analyzed during this study are included in this published article and its additional files.

\section{Consent for publication}

Not applicable.

\section{Funding}

This work was supported by the Tunisian Ministry of Health (PEC-4-TUN), the Tunisian Ministry of Higher Education and Scientific Research (LR11IPT05 and LR16IPT05) and by the E.C. Grant Agreement No 295097 for FP7 project GM-NCD-Inco. MBR is recipient of a Mobidoc Post-Doc Fellowship under the Programme d'Appui au Système de recherche et d'Innovation (PASRI-Europe Aid). The funders had no role in the study design, data collection and analysis, decision to publish, or preparation of the manuscript.

\section{Acknowledgements}

The authors are very grateful to the patients and their families whose participation made this work possible.

\section{References}

1. Ferlay J, Soerjomataram I, Dikshit R, Eser S, Mathers C, Rebelo M, et al. Cancer incidence and mortality worldwide: sources, methods and major patterns in GLOBOCAN 2012. International journal of cancer Journal international du cancer. 2015;136:E359-86.

2. Chouchane L, Boussen H, Sastry KS. Breast cancer in Arab populations: molecular characteristics and disease management implications. The Lancet Oncology. 2013;14:e417-24.

3. Kuchenbaecker KB, Hopper JL, Barnes DR, Phillips KA, Mooij TM, Roos-Blom MJ, et al. Risks of Breast, Ovarian, and Contralateral Breast Cancer for BRCA1 and BRCA2 Mutation Carriers. Jama. 2017;317:2402-16.

4. Grindedal EM, Heramb C, Karsrud I, Ariansen SL, Maehle L, Undlien DE, et al. Current guidelines for BRCA testing of breast cancer patients are insufficient to detect all mutation carriers. BMC cancer. 2017;17:438.

5. Corsini C, Henouda S, Nejima DB, Bertet H, Toledano A, Boussen H, et al. Early onset breast cancer: differences in risk factors, tumor phenotype, and genotype between North African and South European women. Breast cancer research and treatment. 2017;166:631-39.

6. Mavaddat N, Antoniou AC, Easton DF, Garcia-Closas M. Genetic susceptibility to breast cancer. Molecular oncology. 2010;4:174-91.

7. Milne RL, Antoniou AC. Modifiers of breast and ovarian cancer risks for BRCA1 and BRCA2 mutation carriers. Endocrine-related cancer. 2016;23:T69-84.

8. Antoniou AC, Kuchenbaecker KB, Soucy P, Beesley J, Chen X, McGuffog L, et al. Common variants at 12p11, 12q24, 9p21, 9q31.2 and in ZNF365 are associated with breast cancer risk for BRCA1 and/or BRCA2 mutation carriers. Breast cancer research : BCR. 2012;14:R33.

9. Antoniou AC, Spurdle AB, Sinilnikova OM, Healey S, Pooley KA, Schmutzler RK, et al. Common breast cancer-predisposition alleles are associated with breast cancer risk in BRCA1 and BRCA2 mutation carriers. American journal of human genetics. 2008;82:937-48.

10. Antoniou AC, Sinilnikova OM, McGuffog L, Healey S, Nevanlinna H, Heikkinen T, et al. Common variants in LSP1, 2q35 and 8q24 and breast cancer risk for BRCA1 and BRCA2 mutation carriers. Human molecular genetics. 2009;18:4442-56.

11. Gaudet MM, Kuchenbaecker KB, Vijai J, Klein RJ, Kirchhoff T, McGuffog L, et al. Identification of a BRCA2-specific modifier locus at $6 p 24$ related to breast cancer risk. PLoS genetics. 2013;9:e1003173. 
12. Gaudet MM, Kirchhoff T, Green T, Vijai J, Korn JM, Guiducci C, et al. Common genetic variants and modification of penetrance of BRCA2-associated breast cancer. PLoS genetics. 2010;6:e1001183.

13. Maxwell KN, Nathanson KL. Common breast cancer risk variants in the post-COGS era: a comprehensive review. Breast cancer research : BCR. 2013;15:212.

14. Osorio A, Milne RL, Kuchenbaecker K, Vaclova T, Pita G, Alonso R, et al. DNA glycosylases involved in base excision repair may be associated with cancer risk in BRCA1 and BRCA2 mutation carriers. PLoS genetics. 2014;10:e1004256.

15. Benitez-Buelga C, Vaclova T, Ferreira S, Urioste M, Inglada-Perez L, Soberon N, et al. Molecular insights into the OGG1 gene, a cancer risk modifier in BRCA1 and BRCA2 mutations carriers. Oncotarget. 2016;7:25815-25.

16. Antoniou AC, Easton DF. Polygenic inheritance of breast cancer: Implications for design of association studies. Genetic epidemiology. 2003;25:190-202.

17. Pharoah PD, Antoniou A, Bobrow M, Zimmern RL, Easton DF, Ponder BA. Polygenic susceptibility to breast cancer and implications for prevention. Nature genetics. 2002;31:33-6.

18. Mavaddat N, Pharoah PD, Michailidou K, Tyrer J, Brook MN, Bolla MK, et al. Prediction of breast cancer risk based on profiling with common genetic variants. Journal of the National Cancer Institute. 2015;107.

19. Michailidou K, Lindstrom S, Dennis J, Beesley J, Hui S, Kar S, et al. Association analysis identifies 65 new breast cancer risk loci. Nature. 2017;551:92-94.

20. Milne RL, Kuchenbaecker KB, Michailidou K, Beesley J, Kar S, Lindstrom S, et al. Identification of ten variants associated with risk of estrogen-receptornegative breast cancer. Nature genetics. 2017;49:1767-78.

21. Lilyquist J, Ruddy KJ, Vachon CM, Couch FJ. Common Genetic Variation and Breast Cancer Risk - Past, present, and future. Cancer epidemiology, biomarkers \& prevention : a publication of the American Association for Cancer Research, cosponsored by the American Society of Preventive Oncology. 2018.

22. Milne RL, Antoniou AC. Genetic modifiers of cancer risk for BRCA1 and BRCA2 mutation carriers. Annals of oncology : official journal of the European Society for Medical Oncology. 2011;22 Suppl 1:i11-7.

23. Stafford JL, Dyson G, Levin NK, Chaudhry S, Rosati R, Kalpage H, et al. Reanalysis of BRCA1/2 negative high risk ovarian cancer patients reveals novel germline risk loci and insights into missing heritability. PloS one. 2017;12:e0178450.

24. Banerjee T, Brosh RM, Jr. RECQL: a new breast cancer susceptibility gene. Cell cycle (Georgetown, Tex). 2015;14:3540-3.

25. Sokolenko AP, Suspitsin EN, Kuligina E, Bizin IV, Frishman D, Imyanitov EN. Identification of novel hereditary cancer genes by whole exome sequencing. Cancer letters. 2015;369:274-88.

26. Nguyen-Dumont T, Teo ZL, Hammet F, Roberge A, Mahmoodi M, Tsimiklis H, et al. Is RNASEL:p.Glu265* a modifier of early-onset breast cancer risk for carriers of high-risk mutations? BMC cancer. 2018;18:165.

27. Stelzer G, Plaschkes I, Oz-Levi D, Alkelai A, Olender T, Zimmerman S, et al. VarElect: the phenotype-based variation prioritizer of the GeneCards Suite. BMC genomics. 2016;17 Suppl 2:444.

28. Johnson N, Fletcher O, Palles C, Rudd M, Webb E, Sellick G, et al. Counting potentially functional variants in BRCA1, BRCA2 and ATM predicts breast cancer susceptibility. Human molecular genetics. 2007;16:1051-7.

29. Garre P, Martin L, Sanz J, Romero A, Tosar A, Bando I, et al. BRCA2 gene: a candidate for clinical testing in familial colorectal cancer type X. Clinical genetics. 2015;87:582-7.

30. Thomassen M, Hansen TV, Borg A, Lianee HT, Wikman F, Pedersen IS, et al. BRCA1 and BRCA2 mutations in Danish families with hereditary breast and/or ovarian cancer. Acta oncologica (Stockholm, Sweden). 2008;47:772-7.

31. Moller P, Hagen Al, Apold J, Maehle L, Clark N, Fiane B, et al. Genetic epidemiology of BRCA mutations-family history detects less than $50 \%$ of the mutation carriers. European journal of cancer (Oxford, England : 1990). 2007;43:1713-7.

32. Woodage T, King SM, Wacholder S, Hartge P, Struewing JP, McAdams M, et al. The APCI1307K allele and cancer risk in a community-based study of Ashkenazi Jews. Nature genetics. 1998;20:62-5.

33. Redston M, Nathanson KL, Yuan ZQ, Neuhausen SL, Satagopan J, Wong N, et al. The APCI1307K allele and breast cancer risk. Nature genetics. 1998;20:13-4.

34. Leshno A, Shapira S, Liberman E, Kraus S, Sror M, Harlap-Gat A, et al. The APC I1307K allele conveys a significant increased risk for cancer. International journal of cancer Journal international du cancer. 2016;138:1361-7.

35. Abdel-Malak C, Darwish H, Elsaid A, El-Tarapely F, Elshazli R. Association of APC 11307K and E1317Q polymorphisms with colorectal cancer among Egyptian subjects. Familial cancer. 2016;15:49-56.

36. Kapitanovic S, Cacev T, Radosevic S, Spaventi S, Spaventi R, Pavelic K. APC gene loss of heterozygosity, mutations, E1317Q, and I1307K germ-line variants in sporadic colon cancer in Croatia. Experimental and molecular pathology. 2004;77:193-200.

37. Liang J, Lin C, Hu F, Wang F, Zhu L, Yao X, et al. APC polymorphisms and the risk of colorectal neoplasia: a HuGE review and meta-analysis. American journal of epidemiology. 2013;177:1169-79.

38. Alloisio N, Wilmotte R, Morle L, Baklouti F, Marechal J, Ducluzeau MT, et al. Spectrin Jendouba: an alpha II/31 spectrin variant that is associated with elliptocytosis and carries a mutation distant from the dimer self-association site. Blood. 1992;80:809-15.

39. Niss O, Chonat S, Dagaonkar N, Almansoori MO, Kerr K, Rogers ZR, et al. Genotype-phenotype correlations in hereditary elliptocytosis and hereditary pyropoikilocytosis. Blood cells, molecules \& diseases. 2016;61:4-9. 
40. Mencacci NE, Isaias IU, Reich MM, Ganos C, Plagnol V, Polke JM, et al. Parkinson's disease in GTP cyclohydrolase 1 mutation carriers. Brain : a journal of neurology. 2014;137:2480-92.

41. Audebert M, Chevillard S, Levalois C, Gyapay G, Vieillefond A, Klijanienko J, et al. Alterations of the DNA repair gene OGG1 in human clear cell carcinomas of the kidney. Cancer research. 2000;60:4740-4.

42. Dumanski JP, Rasi C, Bjorklund P, Davies H, Ali AS, Gronberg M, et al. A MUTYH germline mutation is associated with small intestinal neuroendocrine tumors. Endocrine-related cancer. 2017;24:427-43.

43. Hager J, Hansen L, Vaisse C, Vionnet N, Philippi A, Poller W, et al. A missense mutation in the glucagon receptor gene is associated with non-insulindependent diabetes mellitus. Nature genetics. 1995;9:299-304.

44. Brand E, Bankir L, Plouin PF, Soubrier F. Glucagon receptor gene mutation (Gly40Ser) in human essential hypertension: the PEGASE study. Hypertension (Dallas, Tex : 1979). 1999;34:15-7.

45. Strazzullo P, lacone R, Siani A, Barba G, Russo O, Russo P, et al. Altered renal sodium handling and hypertension in men carrying the glucagon receptor gene (Gly40Ser) variant. Journal of molecular medicine (Berlin, Germany). 2001;79:574-80.

46. Yagi T, Kubota E, Koyama H, Tanaka T, Kataoka H, Imaeda K, et al. Glucagon promotes colon cancer cell growth via regulating AMPK and MAPK pathways. Oncotarget. 2018;9:10650-64.

47. Zhou Y, Zhang X, Gu C, Xia J. Influence of diabetes mellitus on mortality in breast cancer patients. ANZ journal of surgery. 2015;85:972-8.

48. Tio M, Andrici J, Eslick GD. Folate intake and the risk of breast cancer: a systematic review and meta-analysis. Breast cancer research and treatment. 2014;145:513-24.

49. Syrjakoski K, Kuukasjarvi T, Waltering K, Haraldsson K, Auvinen A, Borg A, et al. BRCA2 mutations in 154 finnish male breast cancer patients. Neoplasia (New York, NY). 2004;6:541-5.

50. Sugano K, Nakamura S, Ando J, Takayama S, Kamata H, Sekiguchi I, et al. Cross-sectional analysis of germline BRCA1 and BRCA2 mutations in Japanese patients suspected to have hereditary breast/ovarian cancer. Cancer science. 2008;99:1967-76.

51. Narayan S, Jaiswal AS, Law BK, Kamal MA, Sharma AK, Hromas RA. Interaction between APC and Fen1 during breast carcinogenesis. DNA repair. 2016;41:54-62.

52. Jaiswal AS, Narayan S. A novel function of adenomatous polyposis coli (APC) in regulating DNA repair. Cancer letters. 2008;271:272-80.

53. Van der Auwera I, Van Laere SJ, Van den Bosch SM, Van den Eynden GG, Trinh BX, van Dam PA, et al. Aberrant methylation of the Adenomatous Polyposis Coli (APC) gene promoter is associated with the inflammatory breast cancer phenotype. British journal of cancer. 2008;99:1735-42.

54. Imaoka T, Okamoto M, Nishimura M, Nishimura Y, Ootawara M, Kakinuma S, et al. Mammary tumorigenesis in ApcMin/+ mice is enhanced by X irradiation with a characteristic age dependence. Radiation research. 2006;165:165-73.

55. VanKlompenberg MK, Bedalov CO, Soto KF, Prosperi JR. APC selectively mediates response to chemotherapeutic agents in breast cancer. BMC cancer. 2015;15:457.

56. Das D, Preet R, Mohapatra P, Satapathy SR, Siddharth S, Tamir T, et al. 5-Fluorouracil mediated anti-cancer activity in colon cancer cells is through the induction of Adenomatous Polyposis Coli: Implication of the long-patch base excision repair pathway. DNA repair. 2014;24:15-25.

57. Ali K, Mahjabeen I, Sabir M, Mehmood H, Kayani MA. OGG1 Mutations and Risk of Female Breast Cancer: Meta-Analysis and Experimental Data. Disease markers. 2015;2015:690878.

58. Benitez-Buelga C, Baquero JM, Vaclova T, Fernandez V, Martin P, Inglada-Perez L, et al. Genetic variation in the NEIL2 DNA glycosylase gene is associated with oxidative DNA damage in BRCA2 mutation carriers. Oncotarget. 2017;8:114626-36.

59. Mazoyer S, Dunning AM, Serova O, Dearden J, Puget N, Healey CS, et al. A polymorphic stop codon in BRCA2. Nature genetics. 1996;14:253-4.

60. Li Q, Guan R, Qiao Y, Liu C, He N, Zhang X, et al. Association between the BRCA2 rs144848 polymorphism and cancer susceptibility: a meta-analysis. Oncotarget. 2017;8:39818-32.

61. Wu K, Hinson SR, Ohashi A, Farrugia D, Wendt P, Tavtigian SV, et al. Functional evaluation and cancer risk assessment of BRCA2 unclassified variants. Cancer research. 2005;65:417-26.

62. Meeks HD, Song H, Michailidou K, Bolla MK, Dennis J, Wang Q, et al. BRCA2 Polymorphic Stop Codon K3326X and the Risk of Breast, Prostate, and Ovarian Cancers. Journal of the National Cancer Institute. 2016;108.

63. Delahaye-Sourdeix M, Anantharaman D, Timofeeva MN, Gaborieau V, Chabrier A, Vallee MP, et al. A rare truncating BRCA2 variant and genetic susceptibility to upper aerodigestive tract cancer. Journal of the National Cancer Institute. 2015;107.

64. Ge Y, Wang Y, Shao W, Jin J, Du M, Ma G, et al. Rare variants in BRCA2 and CHEK2 are associated with the risk of urinary tract cancers. Scientific reports. 2016;6:33542.

65. de Garibay GR, Acedo A, Garcia-Casado Z, Gutierrez-Enriquez S, Tosar A, Romero A, et al. Capillary electrophoresis analysis of conventional splicing assays: IARC analytical and clinical classification of 31 BRCA2 genetic variants. Human mutation. 2014;35:53-7.

66. McAllister KA, Bennett LM, Houle CD, Ward T, Malphurs J, Collins NK, et al. Cancer susceptibility of mice with a homozygous deletion in the COOH-terminal domain of the Brca2 gene. Cancer research. 2002;62:990-4.

67. Atanassov BS, Barrett JC, Davis BJ. Homozygous germ line mutation in exon 27 of murine Brca2 disrupts the Fancd2-Brca2 pathway in the homologous recombination-mediated DNA interstrand cross-links' repair but does not affect meiosis. Genes, chromosomes \& cancer. 2005;44:429-37.

68. Quiroz-Zarate A, Harshfield BJ, Hu R, Knoblauch N, Beck AH, Hankinson SE, et al. Expression Quantitative Trait loci (QTL) in tumor adjacent normal breast tissue and breast tumor tissue. PloS one. 2017;12:e0170181. 
69. Hamdi Y, Soucy P, Adoue V, Michailidou K, Canisius S, Lemacon A, et al. Association of breast cancer risk with genetic variants showing differential allelic expression: Identification of a novel breast cancer susceptibility locus at 4q21. Oncotarget. 2016;7:80140-63.

70. Hamdi Y, Ben Rekaya M, Jingxuan S, Nagara M, Messaoud O, Benammar Elgaaied A, et al. A genome wide SNP genotyping study in the Tunisian population: specific reporting on a subset of common breast cancer risk loci. BMC cancer. 2018;18:1295.

71. Bodmer W, Tomlinson I. Rare genetic variants and the risk of cancer. Current opinion in genetics \& development. 2010;20:262-7.

72. Garrett LT, Hickman N, Jacobson A, Bennett RL, Amendola LM, Rosenthal EA, et al. Family Studies for Classification of Variants of Uncertain Classification: Current Laboratory Clinical Practice and a New Web-Based Educational Tool. Journal of genetic counseling. 2016;25:1146-56.

73. Moghadasi S, Meeks HD, Vreeswijk MP, Janssen LA, Borg A, Ehrencrona H, et al. The BRCA1 c. 5096G>A p.Arg1699GIn (R1699Q) intermediate risk variant: breast and ovarian cancer risk estimation and recommendations for clinical management from the ENIGMA consortium. Journal of medical genetics. 2018;55:15-20.

74. Ben Halim N, Ben Alaya Bouafif N, Romdhane L, Kefi Ben Atig R, Chouchane I, Bouyacoub Y, et al. Consanguinity, endogamy, and genetic disorders in Tunisia. Journal of community genetics. 2013;4:273-84.

75. Romdhane L, Ben Halim N, Rejeb I, Kefi R, Bouyacoub Y, Ben Rekaya M, et al. Specific aspects of consanguinity: some examples from the tunisian population. Human heredity. 2014;77:167-74.

76. Jerbi M, Ben Rekaya M, Naouali C, Jones M, Messaoud O, Tounsi H, et al. Clinical, genealogical and molecular investigation of the xeroderma pigmentosum type $\mathrm{C}$ complementation group in Tunisia. The British journal of dermatology. 2016;174:439-43.

77. Amouri A, Talmoudi F, Messaoud O, d'Enghien CD, Rekaya MB, Allegui I, et al. High frequency of exon 15 deletion in the FANCA gene in Tunisian patients affected with Fanconi anemia disease: implication for diagnosis. Molecular genetics \& genomic medicine. 2014;2:160-5.

\section{Tables}

Table 1: Epidemiological and clinical features of breast cancer affected family members. 


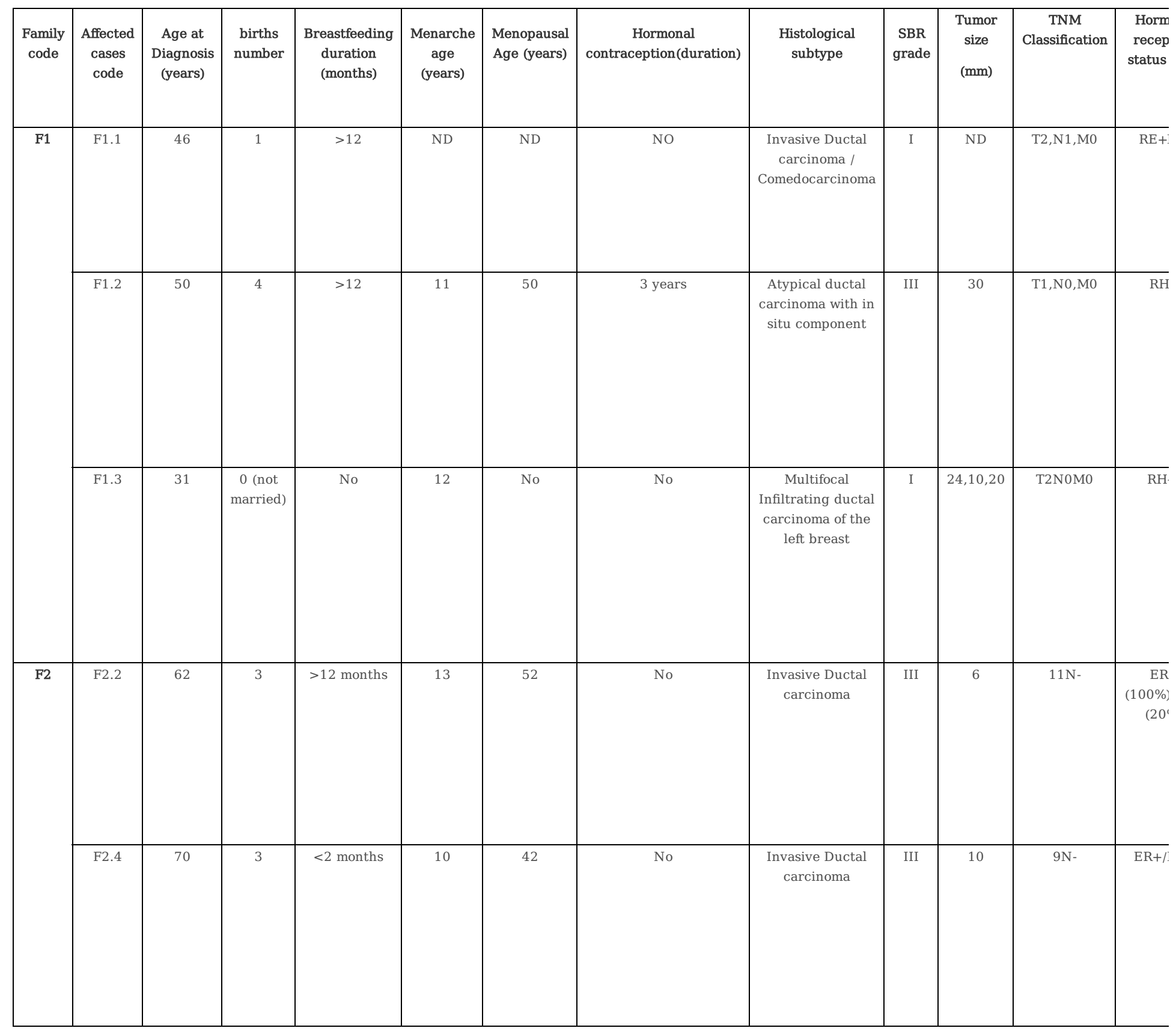

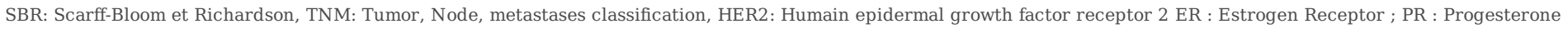
Receptor ; HT : Hormonal Therapy ; ND: Not determined

\section{Table 2: List of all rare and pathogenic variants found in F1.1 and F2.2}




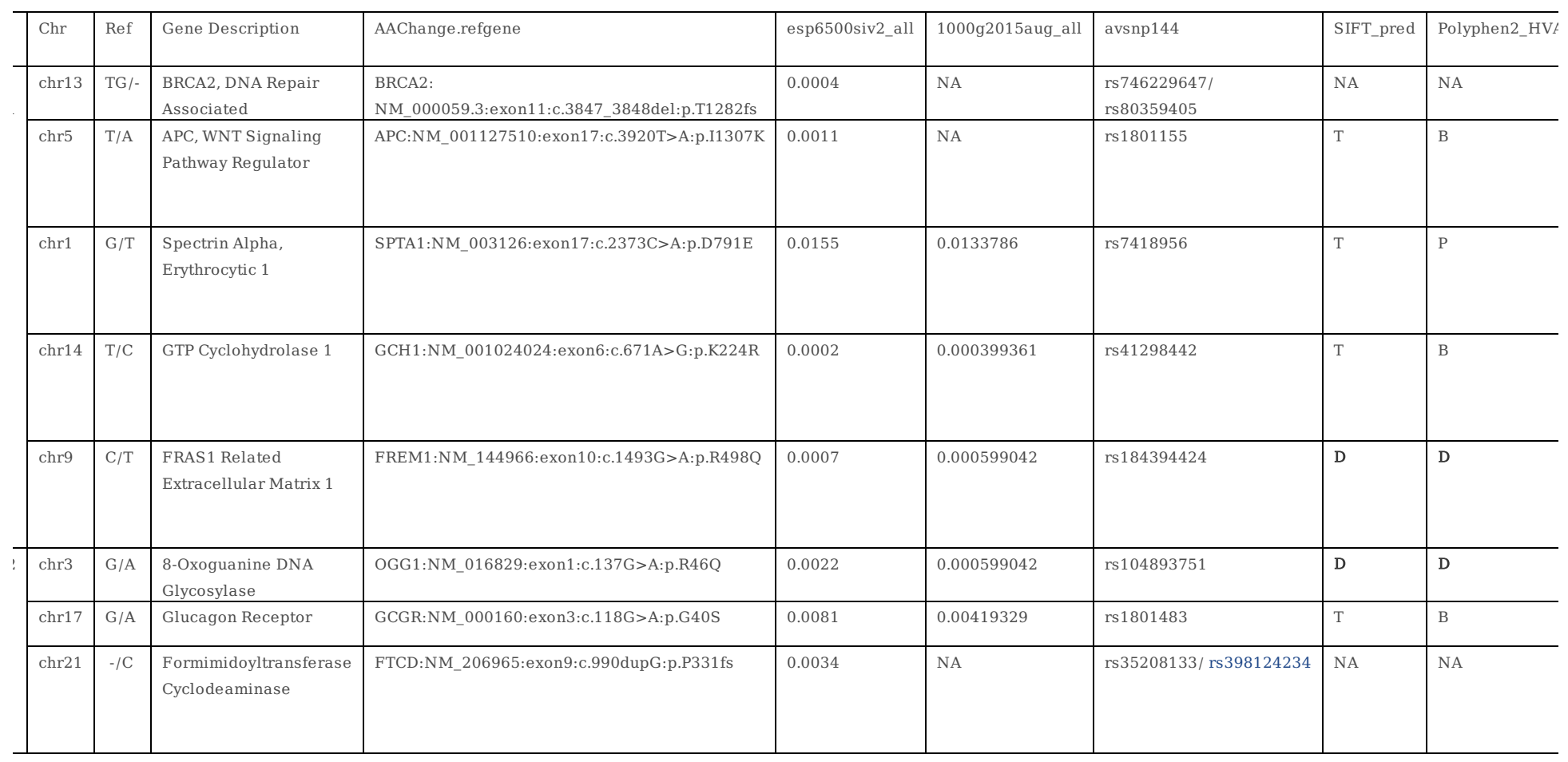

Variant in red are confirmed by Sanger sequencing

Table 3: Variants in BRCA1 and BRCA2 genes in F1.1 and F2.2 patients and their functional annotations. 


\begin{tabular}{|c|c|c|c|c|c|c|c|c|c|c|}
\hline & Gene & avsnp144 & Ref & Alt & Genotype & Func & ExonicFunc & AAChange & 1000 genomes & $\overline{E x}$ \\
\hline \multirow[t]{12}{*}{ F1.1 } & \multirow[t]{6}{*}{ BRCA1 } & rs799917 & G & A & het & exonic & Misence & NM_007300:exon10:c.2612C>T:p.P871L & 0.54393 & $\overline{0.41}$ \\
\hline & & rs368080376 & AAAT & - & het & intronic & NA & NA & 0.000798722 & $\mathrm{~N} /$ \\
\hline & & rs799916 & $\mathrm{T}$ & G & het & intronic & NA & NA & 0.502396 & $\overline{\mathrm{N} t}$ \\
\hline & & rs368252296/rs80308573 & $\mathrm{A}$ & - & het & intronic & NA & NA & NA & $\overline{\mathrm{N}} \mathrm{t}$ \\
\hline & & rs799905 & G & $\mathrm{C}$ & het & intronic & NA & NA & 0.545128 & $\overline{0.4 \varepsilon}$ \\
\hline & & rs80358329 & AGA & - & het & exonic & $\begin{array}{c}\text { nonframeshift } \\
\text { deletion }\end{array}$ & NM_007300:exon10:c.1846_1848del:p.616_616del & 0.00119808 & 0.00 \\
\hline & \multirow[t]{6}{*}{ BRCA2 } & rs543304 & $\mathrm{T}$ & $\mathrm{C}$ & het & exonic & synSNV & BRCA2:NM_000059:exon11:c.3807T>C:p.V1269V & 0.168131 & $\overline{0.18}$ \\
\hline & & rs169547 & $\mathrm{T}$ & $\mathrm{C}$ & het & exonic & Misence & BRCA2:NM_000059:exon14:c.7397T>C:p.V2466A & 0.975839 & $\overline{0.99}$ \\
\hline & & rs11571744 & $\mathrm{C}$ & $\mathrm{T}$ & het & intronic & NA & NA & 0.0161741 & 0.00 \\
\hline & & rs206076 & G & $\mathrm{C}$ & het & exonic & synSNV & BRCA2:NM_000059:exon11:c.6513G>C:p.V2171V & 0.973642 & 0.99 \\
\hline & & rs80359405/rs746229647 & TG & - & het & exonic & $\begin{array}{c}\text { frameshift } \\
\text { deletion }\end{array}$ & BRCA2:NM_000059:exon11:c.3846_3847del:p.T1282fs & NA & $\overline{0.0 \mathrm{C}}$ \\
\hline & & rs206075 & A & G & het & exonic & synSNV & BRCA2:NM_000059:exon11:c.4563A>G:p.L1521L & 0.974042 & $\overline{0.99}$ \\
\hline \multirow[t]{26}{*}{ F2.2 } & \multirow[t]{17}{*}{ BRCA1 } & rs273902772 & $\mathrm{A}$ & - & het & intronic & NA & NA & 0.334864 & $\overline{\mathrm{N} /}$ \\
\hline & & rs3765640 & A & G & hom & intronic & NA & NA & 0.353634 & $\mathbf{N}$ \\
\hline & & rs8176233 & $\mathrm{T}$ & $\mathrm{C}$ & het & intronic & NA & NA & 0.354633 & $\overline{\mathrm{N} /}$ \\
\hline & & rs799917 & G & A & het & exonic & Misence & NM_007300:exon10:c.2612C>T:p.P871L & 0.54393 & 0.41 \\
\hline & & rs1060915 & $\mathbf{A}$ & G & het & exonic & synSNV & NM_007300:exon12:c.4308T>C:p.S1436S & 0.336262 & 0.34 \\
\hline & & rs16941 & $\mathrm{T}$ & $\mathrm{C}$ & het & exonic & Misence & NM_007300:exon10:c.3113A>G:p.E1038G & 0.335663 & 0.34 \\
\hline & & rs799905 & G & $\mathrm{C}$ & het & intronic & NA & NA & 0.545128 & $0.4 \varepsilon$ \\
\hline & & rs1799949 & G & A & het & exonic & synSNV & NM_007300:exon10:c.2082C>T:p.S694S & 0.336462 & 0.34 \\
\hline & & rs1799965 & $\mathrm{G}$ & $\mathrm{A}$ & het & exonic;splicing & synSNV & NM_007300:exon8:c.591C>T:p.C197C & 0.000399361 & 0.00 \\
\hline & & rs273900734/rs8176212 & $\mathrm{G}$ & $\mathrm{C}$ & het & intronic & NA & NA & 0.353435 & $\overline{\mathrm{N}} \mathrm{s}$ \\
\hline & & rs8176234 & $\mathrm{T}$ & $\mathrm{C}$ & het & intronic & NA & NA & 0.354633 & $\mathrm{~N} /$ \\
\hline & & rs16942 ${ }^{a}$ & $\mathrm{~T}$ & $\mathrm{C}$ & het & exonic & Misence & NM_007300:exon10:c.3548A>G:p.K1183R & 0.352636 & 0.34 \\
\hline & & rs3092994 & $\mathrm{C}$ & $\mathrm{T}$ & het & intronic & NA & NA & 0.342452 & $\mathrm{~N} /$ \\
\hline & & rs1799966 & $\mathrm{T}$ & C & het & exonic & Misence & NM_007300:exon16:c.4900A>G:p.S1634G & 0.355831 & $\overline{0.34}$ \\
\hline & & rs80308573/rs368252296 & $\mathrm{A}$ & - & het & intronic & NA & NA & NA & $\overline{\mathrm{N} t}$ \\
\hline & & rs8176235 & $\mathrm{C}$ & $\mathrm{T}$ & het & intronic & NA & NA & 0.306909 & $\overline{\mathrm{N} / \mathrm{H}}$ \\
\hline & & $\mathrm{rs} 16940^{\mathrm{a}}$ & A & G & het & exonic & synSNV & NM_007300:exon10:c.2311T>C:p.L771L & 0.335264 & 0.34 \\
\hline & \multirow[t]{9}{*}{ BRCA2 } & rs543304 & $\mathrm{T}$ & $\mathrm{C}$ & het & exonic & synSNV & BRCA2:NM_000059:exon11:c.3807T>C:p.V1269V & 0.168131 & 0.18 \\
\hline & & rs206076 & G & $\mathrm{C}$ & hom & exonic & synSNV & BRCA2:NM_000059:exon11:c.6513G>C:p.V2171V & 0.973642 & 0.99 \\
\hline & & rs169547 & $\mathrm{T}$ & C & hom & exonic & Misence & BRCA2:NM_000059:exon14:c.7397T>C:p.V2466A & 0.975839 & 0.99 \\
\hline & & rs11571744 & $\mathrm{C}$ & $\mathrm{T}$ & het & intronic & NA & NA & 0.0161741 & 0.00 \\
\hline & & rs11571833 ${ }^{\mathrm{a}}$ & $\mathrm{A}$ & $\mathrm{T}$ & het & exonic & stopgain & BRCA2:NM_000059:exon27:c.9976A>T:p.K3326X & 0.00439297 & 0.00 \\
\hline & & rs206073 & $\mathrm{G}$ & A & hom & intronic & NA & NA & 0.974042 & $\overline{\mathrm{N} /}$ \\
\hline & & rs144549870 & TATCT & - & het & intronic & NA & NA & 0.0157748 & $\overline{\mathrm{N} /}$ \\
\hline & & $\mathrm{rs} 144848^{\mathrm{a}}$ & A & C & het & exonic & Misence & BRCA2:NM_000059:exon10:c.1114A>C:p.N372H & 0.249401 & 0.27 \\
\hline & & rs206075 & A & G & hom & exonic & synSNV & BRCA2:NM_000059:exon11:c.4563A>G:p.L1521L & 0.974042 & $\overline{0.99}$ \\
\hline
\end{tabular}

Page 13/15 


\begin{tabular}{c|c|c|c|c|c|c|c|c|c|} 
& rs11571818 & $\mathrm{T}$ & $\mathrm{C}$ & het & intronic & NA & NA & 0.00439297 & 0.00 \\
\cline { 2 - 8 } & rs206080 & T & C & hom & intronic & NA & NA & 0.974042 & N $/$ \\
\hline
\end{tabular}

Variant in red are confirmed by Sanger sequencing. (a )Variants previously reported to represent independently minor, but cumulatively significant, increased risk for breast cancer. Variants highlighted in bold present functional effect according to RegulomeDB data

\section{Figures}

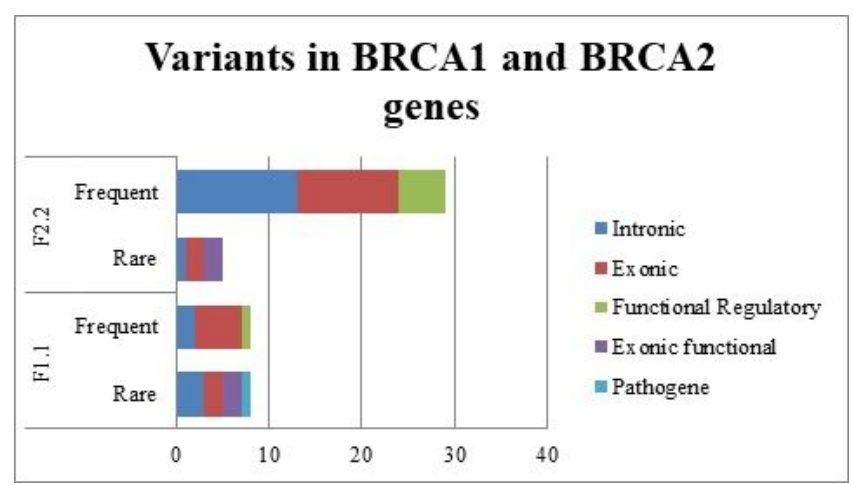

\section{Figure 1}

The structure of genetic profile of BRCA1 and BRCA2 genes in F1.1 and F2.2 patients

F1 Family

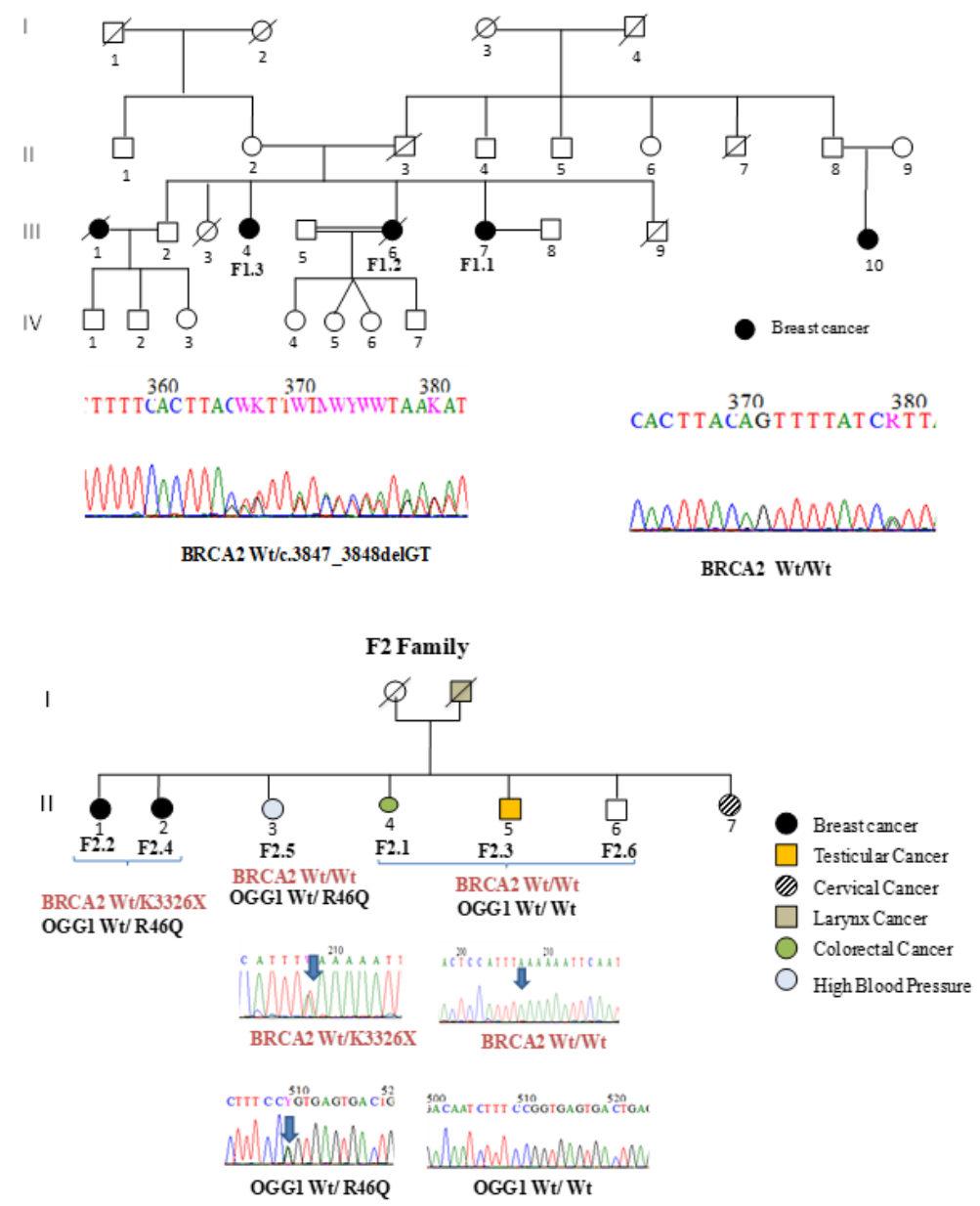

Figure 2

Pedigrees of the F1 and F2 families and DNA-sequence electropherograms for unaffected (wt) and affected family members. 


\section{Supplementary Files}

This is a list of supplementary files associated with this preprint. Click to download.

- supplementarytables1231.pdf 